\title{
Information Criteria for quantifying loss of reversibility in parallelized KMC
}

\author{
Konstantinos Gourgoulias ${ }^{\mathrm{a}, *}$, Markos A. Katsoulakis ${ }^{\mathrm{a}}$, Luc Rey-Bellet ${ }^{\mathrm{a}}$ \\ ${ }^{a}$ Department of Mathematics and Statistics, Lederle Graduate Research Tower, University \\ of Massachusetts, 710 N. Pleasant Street, Amherst, MA, 01003-9305
}

\begin{abstract}
Parallel Kinetic Monte Carlo (KMC) is a potent tool to simulate stochastic particle systems efficiently. However, despite literature on quantifying domain decomposition errors of the particle system for this class of algorithms in the short and in the long time regime, no study yet explores and quantifies the loss of time-reversibility in Parallel KMC. Inspired by concepts from non-equilibrium statistical mechanics, we propose the entropy production per unit time, or entropy production rate, given in terms of an observable and a corresponding estimator, as a metric that quantifies the loss of reversibility. Typically, this is a quantity that cannot be computed explicitly for Parallel KMC, which is why we develop a posteriori estimators that have good scaling properties with respect to the size of the system. Through these estimators, we can connect the different parameters of the scheme, such as the communication time step of the parallelization, the choice of the domain decomposition, and the computational schedule, with its performance in controlling the loss of reversibility. From this point of view, the entropy production rate can be seen both as an information criterion to compare the reversibility of different parallel schemes and as a tool to diagnose reversibility issues with a particular scheme. As a demonstration, we use Sandia Lab's SPPARKS software to compare different parallelization schemes and different domain (lattice) decompositions.
\end{abstract}

Keywords: parallel kinetic Monte Carlo, operator splitting schemes, long-time errors, time-reversibility, detailed balance, entropy production, information criteria

\section{Introduction}

Kinetic Monte Carlo, also known as the n-fold way [1] or the Bortz-KalosLebowitz algorithm [2], is a common tool among practitioners interested in simulating stochastic processes arising from chemical, biological, or agent-based

${ }^{*}$ Corresponding author. e-mail: gourgoul@math.umass.edu

Preprint submitted to Elsevier

September 12, 2016

(C) 2016. This manuscript version is made available under the Elsevier user license http://www.elsevier.com/open-access/userlicense/1.0/ 
models on lattices [3]. However, even sophisticated algorithms inevitably experience slowdown as the size of the system increases. In fact, it may even be the case that the system size prohibits the use of a serial simulation, for instance due to problems with storing the system in a single CPU's memory.

There is a substantial amount of work in addressing the efficiency issues when simulating larger time and length scales by using parallel algorithms for systems with either short-range $[4,5,6]$ or long-range interactions $[7,8]$. Typically, those algorithms are based on domain decomposition of the lattice into subdomains (see Figure 1), and subsequently the simulation on each sub-domain according to a chosen computational schedule. One such algorithm is part of Sandia Labs' SPPARKS Monte Carlo code [9]. A new insight from [10] was that such parallel KMC algorithms that depend on short-range interactions can be formulated as operator splitting schemes that approximate the exact process. This mathematical formulation allows both for performance and numerical error analysis of the schemes [11]. It was also leveraged in previous work [12] where, combined with information metrics, allowed us to study the long time error behavior of the schemes.

In fact, the investigation of long-time errors for operator splitting schemes is of prime importance when using parallel KMC, as errors accumulate due to the domain decomposition procedure. This accumulation can affect the simulation dramatically at long times and make it uncertain for practitioners to sample from the correct stationary regime. Unfortunately, classical numerical analysis fails to quantify errors of splitting schemes, such as parallel KMC for long times, which in turn motivated our use of the relative entropy per unit time as a tool to study the performance of operator splitting schemes [12].

Another aspect of long-time behavior, and the focus of this work, is on systems with time-reversible dynamics. That symmetry is often an integral part of the physical structure of the model, for example in the simulation of interacting diffusions or adsorption/desorption mechanisms. While in such cases the time-reversal symmetry is preserved under the serial KMC simulation (typically by enforcing the detailed balance condition), the time-discretization, domain decomposition, and breakdown of serial communication of the parallelized algorithm may lead to loss of detailed balance, and thus of reversibility. There exists some literature on constructing parallel algorithms that preserve the detailed balance (DB) condition [13]. In those algorithms, the scheme picks a schedule for sweeping over the lattice sub-domains, executes it by simulating each sub-domain forward in time for a fixed number of time steps according to the schedule, and then picks a new schedule. For the adjustment to the correct timescale, computation of an equilibrium autocorrelation function is also required. Although such schemes resemble the random Lie-Trotter splitting [11] and they can be numerically analyzed in a similar manner, we will not discuss them here, mainly due to the technical differences with schemes that employ a fixed computational schedule [10]. More specificially, our focus is on general partially asynchronous parallel algorithms, like the one in SPPARKS. For those, a user has to pick between different domain decompositions, time steps of the scheme, and a fixed schedule. These choices will impact the loss of reversibility 
of the scheme. Therefore, it makes sense to develop a theory that can connect the various parameters of the scheme with loss of reversibility.

Regarding the loss of reversibility of numerical schemes, in [14] the authors used the entropy production rate (EPR) as an information metric to quantify the loss of reversibility for the Euler-Maruyama and Milstein schemes for stochastic differential equations, as well as BBK schemes for Langevin dynamics. This idea was motivated by concepts in non-equilibrium statistical mechanics, originally developed to understand the long-time dynamics and the fluctuations in nonequilibrium steady states $[15,16,17,18,19]$. The authors in [14] used such non-equilibrium statistical mechanics methods as computable numerical tools to assess the loss of reversibility of numerical schemes for SDEs. More specifically, they computed the EPR with the Gallavotti-Cohen action functional [17] as an estimator for different numerical schemes. It was demonstrated that the scheme performance in controlling the loss of reversibility can vary greatly. In particular, the Euler-Maruyama scheme for SDEs with multiplicative noise can break reversibility in an unrecoverable manner regardless of the size of the time step [14, Theorem 3.7].

Our goal here is to apply a similar perspective for the study of splitting schemes in parallel KMC. However, in contrast with schemes for SDEs, for the class of systems that we can simulate in this manner the transition probabilities are either difficult to compute or not available at all. Because of this, a new approach is required, which is why we write the EPR as an asymptotic expansion in the scheme's time step by using the semigroup theory for Markov chains. We demonstrate that the coefficients of the expansion of the EPR depend on the transition rates of the model and can be estimated as ergodic averages by samples from the parallel algorithm. We also show that the required computations for the estimation of the coefficients scale with the size of the boundary between sub-domains on the lattice in a manner that depends on the scheme selected. Therefore, by appropriate normalization, we can calculate the entropy production rate per lattice site, i.e. independent of system size. As a result, we obtain an a posteriori expansion for the estimator of the EPR, which can be used as a diagnostic tool that can be calculated on a system of smaller size than the targeted one, and/or even ran with a simple serial implementation of the parallel algorithm.

This information-theoretical perspective is similar to the use of information metrics to assess discrepancy of models, algorithms, approximations, etc., to that applied to the study of long time errors for Parallel KMC [12], sensitivity analysis [20], and in studying loss of information due to coarse-graining in nonequilibrium systems [21].

The manuscript is organized as follows. In Section 2, we provide an introduction to Parallel Lattice KMC and the ideas behind its error analysis based on operator splitting. Section 3 is especially important, as we introduce the entropy production and entropy production per unit time. Those concepts will be the information-theoretical quantities used to study loss of reversibility for operator splitting schemes. Then, in Section 4, we discuss the estimation of the EPR, referring to specific examples and an implementation in SPPARKS. We 
use the estimates to compare two splitting schemes as well as discuss their loss of reversibility with respect to lattice decomposition and time step. Finally, in Section 5, we provide general results for the asymptotic behavior of EPR, and deduce from them estimators.

\section{Background on Parallel Lattice KMC}

Parallel Lattice KMC is an approximation to the exact, but serial, simulation algorithm. In implementations, it works by taking advantage of the spatial dependencies between the different events. For example, in a model with finite range interactions, the spins on two lattice sites can change with no error to the dynamics as long as the two are sufficiently far apart. Therefore, by decomposing the lattice into sub-lattices, we gain an efficient alternative to serial KMC analogous to domain decomposition methods in parallel algorithms for partial differential equations.

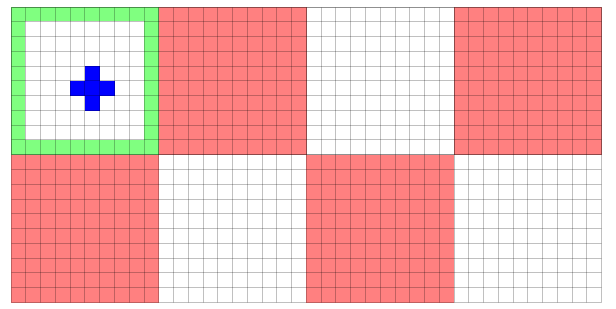

Figure 1: Checkerboard decomposition of a rectangular lattice into sub-lattices. Because each site's transition depends on the information from the nearest neighbors, transitions in sublattices of the same color are independent. White sub-lattices can be simulated asynchronously in time, while keeping the states in the red ones frozen. When the stochastic time reaches $\Delta t$, information is shared with the red sub-lattices about the state of the boundary regions (here only shown for the first sub-lattice).

A new insight provided in [10] was that parallel algorithms, such as the one described in Figure 1, can be formulated as operator splitting schemes. This connection allows for the design, error quantification, and performance analysis of such algorithms [11]. Specifically, this approach allows for an observablefocused error analysis, through which a practitioner can pick both the scheme class and specific parameters that fit the computational needs. Additionally, it formalizes the dependence of the error on the decomposition of the lattice and on the splitting time step, $\Delta t$, for bounded time intervals. Finally, it also allows to study the long-time behavior of the schemes and provides long-time error control in the recent work [12].

To begin, we pick a positive operator splitting time step $\Delta t$. If we were to simulate a Continuous Time Markov Chain (CTMC) via the serial KMC algorithm, then the corresponding transition probability of the process jumping from a state $\sigma$ to a state $\sigma^{\prime}, \sigma, \sigma^{\prime} \in S$, in time $t$ would be

$$
P_{t}\left(\sigma, \sigma^{\prime}\right)=P\left(\sigma_{t}=\sigma^{\prime} \mid \sigma_{0}=\sigma\right)=e^{t L} \delta_{\sigma^{\prime}}(\sigma) .
$$




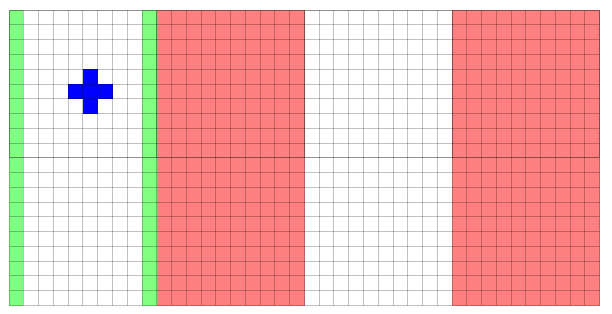

Figure 2: Stripe decomposition of a rectangular lattice into sub-lattices. Compared to Figure 1 , now each processor needs to store more information before the runs can take place.

In (1), $\delta_{\sigma^{\prime}}$ is a Dirac probability measure, centered at state $\sigma^{\prime}$, and $L$ is the generator of the process which, for bounded and continuous functions $f$, is defined as

$$
L[f](\sigma):=\sum_{\sigma^{\prime} \in S} q\left(\sigma, \sigma^{\prime}\right)\left(f\left(\sigma^{\prime}\right)-f(\sigma)\right) .
$$

The transition rates of the CTMC will be denoted by $q(\cdot, \cdot)$. In general, they are tied to the system being modelled and are assumed to be known, see Appendix $\mathrm{C}$ and Appendix D.

Since the approximate process will be a discretization with $\Delta t$ step size, we will be comparing it against the $\Delta t$-skeleton of the exact Continuous Time Markov Chain, with transition probability $P_{\Delta t}\left(\sigma, \sigma^{\prime}\right)=e^{\Delta t L} \delta_{\sigma^{\prime}}(\sigma)$. This is only done to simplify the comparison and corresponds to sub-sampling the exact KMC, keeping only the states every $\Delta t$ apart. Now, inspired by the Trotter product formula [22], one can write approximations to $e^{\Delta t L}$ by splitting the operator $L$ into $L_{1}+L_{2}$ (with associated rates $q_{1}, q_{2}$ ). For example, two popular approximations are:

$$
\begin{aligned}
e^{\Delta t L} & \simeq e^{\Delta t L_{1}} e^{\Delta t L_{2}}, \\
e^{\Delta t L} & \simeq e^{\Delta t / 2 L_{1}} e^{\Delta t L_{2}} e^{\Delta t / 2 L_{1}} .
\end{aligned}
$$

Throughout this work, we shall be using $Q_{\Delta t}$ to denote the transition probability arising from approximations to $e^{\Delta t L}$. We will also use $\mu_{\Delta t}$ to denote the corresponding stationary measure.

Although we consider a splitting into two operators, $L_{1}, L_{2}$, this is for the convenience of the reader. Occasionally, it is beneficial to split the generator $L$ into more than two parts, as is done in Sandia Labb's SPPARKS code [9], where a $2 D$ simulation decomposes the lattice into four pieces instead of two. However, the error analysis extends naturally to this case. 


\subsection{Local Error Analysis}

Operator splitting approximations are equivalent to specific computational schedules for Parallel Lattice KMC schemes [10]. For example, if we alternate between the red and white groups in Figure 1, allowing each group to run only for $\Delta t$, then that is equivalent to using the Lie splitting (Equation (3)) to approximate $e^{\Delta t L}$. If $L$ is a bounded operator, then we can write the semigroup as a series expansion,

$$
e^{\Delta t L}=\sum_{k=0}^{\infty} \frac{\Delta t^{k}}{k !} L^{k}
$$

where $L^{k}$ stands for the resulting operator after $k$ compositions of $L$. We can also write a representation for the various operator splitting schemes. For example, for the case of the Lie splitting in (3) and by using the expansion in (5),

$$
\begin{aligned}
e^{\Delta t L_{1}} e^{\Delta t L_{2}} & =\left(I+\Delta t L_{1}+O\left(\Delta t^{3}\right)\right) \cdot\left(I+\Delta t L_{2}+O\left(\Delta t^{3}\right)\right) \\
& =I+\Delta t L+\frac{\Delta t^{2}}{2}\left(L_{1}^{2}+L_{2}^{2}+2 L_{1} L_{2}\right)+O\left(\Delta t^{3}\right)
\end{aligned}
$$

Then, the representations in (5) and (6) allow us to study the local error between $P_{\Delta t}$ and $Q_{\Delta t}$ :

$$
P_{\Delta t}\left(\sigma, \sigma^{\prime}\right)-Q_{\Delta t}(\sigma, \sigma)=\frac{\Delta t^{2}}{2}\left[L_{1}, L_{2}\right] \delta_{\sigma^{\prime}}(\sigma)+O\left(\Delta t^{3}\right),
$$

where $\left[L_{1}, L_{2}\right]$ is the Lie bracket of $L_{1}, L_{2}$, and is equal to $L_{1} L_{2}-L_{2} L_{1}$. Similarly, the order of the local error $p$ is equal to 2. Note that $L_{1}, L_{2}$ can be expressed in terms of the transition rates, which implies that $\left[L_{1}, L_{2}\right]$ is computable for any pair of states $\left(\sigma, \sigma^{\prime}\right)$. A generalization of this idea is in Lemma 1.

Lemma 1 (Commutator and Order of Local Error). Let $\sigma, \sigma^{\prime}$ be states, $P_{\Delta t}$ as in Equation (1) and $Q_{\Delta t}$ : approximation of $P_{\Delta t}$ via a splitting scheme. Then, there is a function $C: S \times S \rightarrow \mathbb{R}$ and an integer $p, p>1$, such that

$$
P_{\Delta t}\left(\sigma, \sigma^{\prime}\right)-Q_{\Delta t}\left(\sigma, \sigma^{\prime}\right)=C\left(\sigma, \sigma^{\prime}\right) \Delta t^{p}+O\left(\Delta t^{p+1}\right) .
$$

$C$ will be called the commutator and $p$ is the order of the local error.

Proof. Equation (8) can be derived from the power series representations of $P_{\Delta t}, Q_{\Delta t}$, as $L$ is a bounded operator.

In the context of Parallel KMC, the commutator term $C=C\left(\sigma, \sigma^{\prime}\right)$ captures the error due to mismatches on the boundary regions between the different sublattices [11].

The operating assumption in this work is that all operators are bounded. This allows us to represent the transition probabilities with power series and, 
subsequently, to calculate the form of the commutators and of other quantities of interest (see discussion in Appendix B). However, the present work could also be extended to the case of unbounded operators [23, 24], where alternative representations for the semigroups could be used for the error analysis. We are not handling such cases here, as the Markov generators of stochastic particle systems are bounded operators [11].

\section{Entropy Production Rate: an information criterion for reversibil- ity}

Let us consider a discrete stochastic process $X_{n}, n \in \mathbb{N}$. Then, $X_{n}$ is timereversible if, for any $m \in \mathbb{N}$

$$
p\left(\sigma_{0}, \ldots, \sigma_{m}\right)=p\left(\sigma_{m}, \ldots, \sigma_{0}\right),
$$

where $p\left(\sigma_{0}, \ldots, \sigma_{m}\right)=p\left(X_{0}=\sigma_{0}, \ldots, X_{m}=\sigma_{0}\right), \sigma_{i}$ being states of the process. For stationary Markov processes, the detailed balance condition (DB) is equivalent to time-reversibility [25, Theorem 1.2]. If $X_{n}$ has transition probability $P$ and stationary distribution $\mu$, then $\mathrm{DB}$ requires that for all states $\sigma, \sigma^{\prime} \in S$,

$$
\mu(\sigma) P\left(\sigma, \sigma^{\prime}\right)=\mu\left(\sigma^{\prime}\right) P\left(\sigma^{\prime}, \sigma\right) .
$$

Although the DB condition (10) is a useful analytical tool for the construction of Markov Chains with a specific stationary distribution, we cannot apply it to quantify the loss of reversibility for the systems we are interested in. In our context, $P$ corresponds to the transition probability, $Q_{\Delta t}$, of the scheme, which we do not know explicitly, and $\mu=\mu_{\Delta t}$ would be the stationary distribution associated with the scheme, which we can only access through sampling. In addition, due to the time-discretization, domain decomposition, and asynchronous simulation associated with the operator splitting scheme, we do not expect it to exactly satisfy condition (10). Consider for example the case numerical schemes for SDEs [14], where the approximation can completely break down reversibility. In view of this, we wish to quantify the loss of reversibility and connect it to the parameters of the scheme (lattice decomposition, computation schedule, time step $\Delta t$, etc.). Therefore, we need to look for alternative ways to assess the loss of reversibility of the scheme.

Returning to the definition of time-reversibility in (9) with respect to paths, we introduce an object from information theory, the entropy production (EP) associated with $P$ :

$$
\mathrm{EP}(P)=\sum_{\sigma_{0}, \ldots, \sigma_{m}} p\left(\sigma_{0}, \ldots, \sigma_{m}\right) \log \left(\frac{p\left(\sigma_{0}, \ldots, \sigma_{m}\right)}{p\left(\sigma_{m}, \ldots, \sigma_{0}\right)}\right),
$$

with the sum in Equation (11) being over $S^{m}, S$ is the state space.

The EP is an example of a more general measure of similarity between distributions known as the relative entropy (RE), or Kullback-Leibler divergence [26]. 
Given two probability distributions, $p_{1}, p_{2}$, where $p_{1}$ is absolutely continuous with respect to $p_{2}$, then the RE of $p_{1}$ with respect to $p_{2}$ is defined as

$$
R\left(p_{1} \| p_{2}\right):=\int \log \frac{d p_{1}}{d p_{2}} d p_{1} .
$$

The definition in (12) enjoys the properties of a divergence: $1 . R\left(p_{1} \| p_{2}\right) \geq 0$ (Gibbs' inequality), 2. $R\left(p_{1} \| p_{2}\right)=0$ if and only if $p_{1}=p_{2}, p_{1}-$ a.e. However, $\mathrm{RE}$ is not a metric in the strict sense, as it does not satisfy the triangle inequality and is not symmetric in its arguments.

From the second property of a divergence and (11), we can readily see that

$$
\operatorname{EP}(P)=0 \Leftrightarrow p\left(\sigma_{0}, \ldots, \sigma_{m}\right)=p\left(\sigma_{m}, \ldots, \sigma_{0}\right) .
$$

Therefore, if Equation (13) holds for all $m$, then that implies time-reversibility. It is because of this property of the EP that we will use it as a means to assess and quantify how much a scheme $Q_{\Delta t}$ destroys reversibility. This idea was originally motivated by tools in non-equilibrium statistical mechanics to understand long-time dynamics and fluctuations in associated non-equilibrium steady states $[15,16,17,18,19]$.

Calculating the EP, even for moderate $m$, can be computationally intensive. From the definition in (11) we can derive an entropy rate that is independent of the path length when the initial sampling distribution is the stationary. By the Markov property, we can write the forward and backward path distributions as

$$
\begin{aligned}
& p\left(\sigma_{0}, \ldots, \sigma_{m}\right)=\mu\left(\sigma_{0}\right) P\left(\sigma_{0}, \sigma_{1}\right) \cdots P\left(\sigma_{m-1}, \sigma_{m}\right), \\
& p\left(\sigma_{m}, \ldots, \sigma_{0}\right)=\mu\left(\sigma_{m}\right) P\left(\sigma_{m}, \sigma_{m-1}\right) \cdots P\left(\sigma_{1}, \sigma_{0}\right),
\end{aligned}
$$

where $\mu$ is the corresponding stationary distribution. Then, using (14) in Equation (11) and carrying out the calculations leads to

$$
\operatorname{EP}(P)=m \cdot \sum_{\sigma_{0}, \sigma_{1}} \mu\left(\sigma_{0}\right) P\left(\sigma_{0}, \sigma_{1}\right) \log \left(\frac{P\left(\sigma_{0}, \sigma_{1}\right)}{P\left(\sigma_{1}, \sigma_{0}\right)}\right)=m \cdot \operatorname{EPR}(P) .
$$

A formal statement and proof of (15) can be found in Lemma 4, Appendix A.1. The entropy production rate (EPR) is defined for discrete time Markov processes as

$$
\operatorname{EPR}(P):=\sum_{\sigma, \sigma^{\prime}} \mu(\sigma) P\left(\sigma, \sigma^{\prime}\right) \log \left(\frac{P\left(\sigma, \sigma^{\prime}\right)}{P\left(\sigma^{\prime}, \sigma\right)}\right) .
$$

A more general definition, applicable to continuous-time Markov processes, can also be given, see [14] for an application in quantifying the loss of reversibility for numerical schemes for SDEs.

We will use the EPR to quantify the loss of reversibility of the schemes studied. Given $P$, we can estimate the EPR in (16) by the Gallavotti-Cohen functional (as done in [14]):

$$
\operatorname{EPR}(P)=\lim _{N \rightarrow \infty} \frac{1}{N} \sum_{i=0}^{N} \log \left(\frac{P\left(\sigma_{i}, \sigma_{i+1}\right)}{P\left(\sigma_{i+1}, \sigma_{i}\right)}\right),
$$


where $\left(\sigma_{i}, \sigma_{i+1}\right)$ are sampled according to $\mu(\sigma) P\left(\sigma, \sigma^{\prime}\right)$. The quantity on the right hand side of Equation (17) is thus, under suitable ergodic assumptions, an unbiased statistical estimator of the EPR, following the law of large numbers for Markov chains. For a given scheme $Q_{\Delta t}$ with stationary distribution $\mu_{\Delta t}$, Equation (16) thus becomes:

$$
\operatorname{EPR}\left(Q_{\Delta t}\right):=\frac{1}{\Delta t} \sum_{\sigma, \sigma^{\prime}} \mu_{\Delta t}(\sigma) Q_{\Delta t}\left(\sigma, \sigma^{\prime}\right) \log \left(\frac{Q_{\Delta t}\left(\sigma, \sigma^{\prime}\right)}{Q_{\Delta t}\left(\sigma^{\prime}, \sigma\right)}\right)
$$

Remark 1. In Equation (18), we normalize with the time step $\Delta t$ since the EPR is a quantity defined as "per unit time" (see also Equation (15)). This normalization is also practically important, as we wish to consider comparisons of EPRs for different time-steps $\Delta t$. Finally, the same normalization was considered for the RER in previous work [12, Remark 4.2] and Equation (22).

The EP can also be seen as an information criterion for operator splitting schemes. Consider two schemes, $Q_{\Delta t}^{1}, Q_{\Delta t}^{2}$ that approximate the same exact $P_{\Delta t}$. Then we can use EP to quantify which of the two retains more reversibility per time step. That is, we are also interested in making statements of the form

$$
\operatorname{EP}\left(Q_{\Delta t}^{1}\right) \leq \operatorname{EP}\left(Q_{\Delta t}^{2}\right)
$$

$E P\left(Q_{\Delta t}^{1}\right)-E P\left(Q_{\Delta t}^{2}\right)$ is an information criterion that takes into account loss of reversibility, similarly to how $\mathrm{AIC}$ and $\mathrm{BIC}$ are used to assess the quality of models in statistics $[27,28]$. As the EP is a difficult quantity to compute, we can employ the EPR and Equation (15), and thus have another way to distinguish possible schemes based on their performance in controlling the loss of reversibility. In analogy with Inequality (19), we are interested in the difference

$$
\operatorname{EPR}\left(Q_{\Delta t}^{1}\right)-\operatorname{EPR}\left(Q_{\Delta t}^{2}\right)
$$

Even though we have an abstract representation of $Q_{\Delta t}$ (see Equations (3) and (4)), we cannot calculate $Q_{\Delta t}$ directly. What we do know explicitly are the transition rates of the process. We can leverage this information to construct a series expansion of $Q_{\Delta t}$ around $\Delta t$ where each term depends on the transition rates. Through this, we can build statistical estimators of the highest order terms in an expansion of the EPR. Details about the coefficients and their statistical estimation are in Sections 4, 5. In Figure 4 we demonstrate a comparison of two different parallel KMC schemes, based on these computable a posteriori expansions of EPR.

\section{Loss of reversibility in Parallel KMC}

In this section, we will demonstrate how to use the EPR to quantify and control the loss of reversibility for parallel Kinetic Monte Carlo (P-KMC). We

will also mention details about the implementation of the various observables that are needed in order to estimate EPR. 


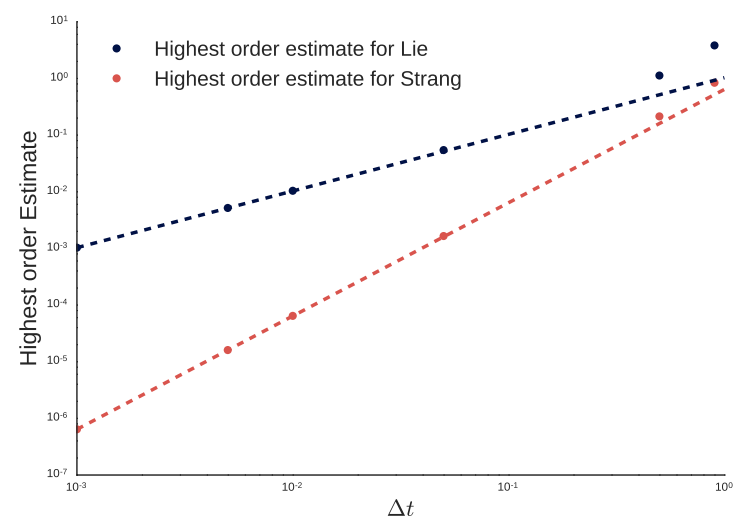

Figure 3: Approximations to the EPR of the form $(A+D) \cdot \Delta t^{p-1}$ in the case of a block decomposition of the lattice. The points depend on $\Delta t$ as we are sampling from different $\mu_{\Delta t}$ to estimate the coefficients, whereas the two dashed curves correspond to $(A+D) \Delta t^{p-1}$ with $A+D$ only estimated at the smallest $\Delta t=0.001$. We use such curves for all comparisons, as we are focused on the case of small $\Delta t$. For $\Delta t$ close to one, the points depart from the line as $\mu_{\Delta t}$ gets further from the exact $\mu_{P}$. The simulated model is an adsorption/desorption system, see Appendix C. The formulas for the coefficients $A$ and $D$ are given in Appendix B. The situation is similar for the decomposition into stripes.

As mentioned before, for stochastic particle dynamics we cannot directly apply the definition in Equation (18), as we do not have the transition probabilities $Q_{\Delta t}$ explicitly. Instead, we will use asymptotic results to approximate the EPR for a small splitting time step, $\Delta t$ (see Section 5 for derivations). We first write the EPR as per Theorem 1, Section 5, but taking also into consideration Remark 1 for the required $\Delta t$ normalization. That is,

$$
\operatorname{EPR}\left(Q_{\Delta t}\right)=H\left(Q_{\Delta t} \mid P_{\Delta t}\right)+I\left(Q_{\Delta t} \mid P_{\Delta t}\right),
$$

where $H$ represents the relative entropy rate (RER)

$$
H\left(Q_{\Delta t} \mid P_{\Delta t}\right):=\frac{1}{\Delta t} \sum_{\sigma, \sigma^{\prime}} \mu_{\Delta t}(\sigma) Q_{\Delta t}\left(\sigma, \sigma^{\prime}\right) \log \left(\frac{Q_{\Delta t}\left(\sigma, \sigma^{\prime}\right)}{P_{\Delta t}\left(\sigma, \sigma^{\prime}\right)}\right)
$$

and $I$ is a "discrepancy" term (see Section 5) defined as

$$
I\left(Q_{\Delta t} \mid P_{\Delta t}\right):=\frac{1}{\Delta t} \sum_{\sigma, \sigma^{\prime}} \mu_{\Delta t}(\sigma) Q_{\Delta t}\left(\sigma, \sigma^{\prime}\right) \log \left(\frac{P_{\Delta t}\left(\sigma^{\prime}, \sigma\right)}{Q_{\Delta t}\left(\sigma^{\prime}, \sigma\right)}\right) .
$$

Before we move on to how results on the RER and $I$ combine to give an asymptotic picture of the EPR, we shall first discuss what each of those captures. The RER, or relative entropy per unit time, has been used in previous work [12] as a means to quantify the long-time error of operator splitting schemes in the 


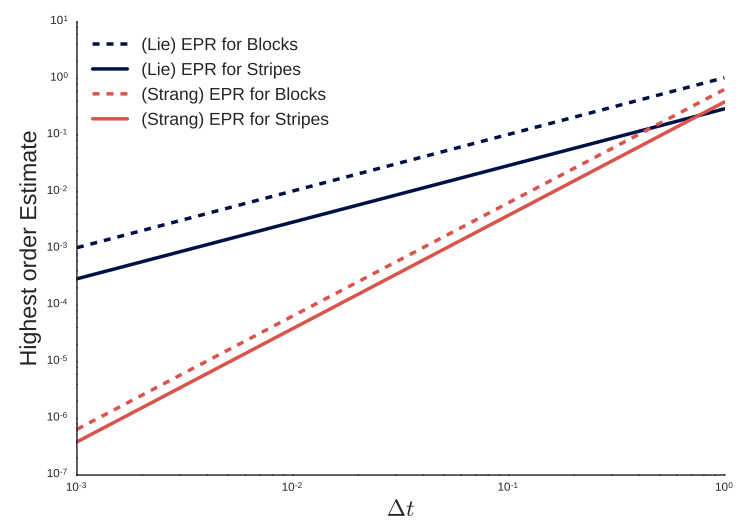

Figure 4: Approximations to the EPR of the form $(A+D) \cdot \Delta t^{p-1}$. The Strang scheme retains more reversibility per time step and is more "stable" (with respect to the entropy production rate) under changes in the decomposition. Also, note that the estimate is normalized by $\Delta t$ as per Remark 1. The example is an adsorption/desorption system, see Appendix C for details on the system and Appendix B for the estimator formulas.

context of parallel KMC. Because of this, the RER can be used as an information criterion to compare such schemes, as it takes into account details of the scheme such as the splitting time step, the domain decomposition of the lattice, and the computational schedule used. The RER has the properties of a divergence, i.e. non-negativity for any $Q_{\Delta t}, P_{\Delta t}$, and equality with zero if and only if $Q_{\Delta t}=P_{\Delta t}$. The discrepancy term in Equation (23) is what enforces the property of the EPR to be zero when $Q_{\Delta t}$ is time-reversible. As we shall see in Section $5, I$ is not a divergence.

Now, by the individual results for the asymptotic behavior of RER (see proof of Theorem 8.6 in [12]) and $I$ (see Equation (41)) for small $\Delta t$, we have

$$
\begin{aligned}
H\left(Q_{\Delta t} \mid P_{\Delta t}\right) & =A \cdot \Delta t^{p-1}+O\left(\Delta t^{p}\right), \\
I\left(Q_{\Delta t} \mid P_{\Delta t}\right) & =D \cdot \Delta t^{p-1}+O\left(\Delta t^{p}\right) .
\end{aligned}
$$

Therefore, from Equations (21), (24), and (25), we get

$$
\operatorname{EPR}\left(Q_{\Delta t}\right)=(A+D) \Delta t^{p-1}+O\left(\Delta t^{p}\right)
$$

We remind here that $p$ stands for the order of the local error (see Lemma 1).

Coefficients $A$ and $D$ are expected values of specific observables with respect to $\mu_{\Delta t}$ (see Appendix C for the explicit formulas in the case of an adsorption/desorption process and Appendix D for the case of a diffusion process). Therefore, under some ergodicity assumptions, they can be estimated via simulation of the system by using the parallel algorithm. In Figures 4 and 5 , we estimate the EPR by an estimation of the constants $A, D$ for small timestep $\Delta t$. 
In previous work [12], we expressed $A$ explicitly in terms of the commutator $C$ and the transition rates of the original process. For example, given a lattice $\Lambda$, for the Lie splitting and an adsorption/desorption example (see Appendix C), the highest order coefficient for the RER is:

$$
\begin{aligned}
A=A_{\text {Lie }} & =\mathbb{E}_{\mu_{\text {Lie }}}\left[\sum_{x, y \in \Lambda} C_{\text {Lie }}\left(\sigma, \sigma^{x, y}\right) F_{\text {Lie }}\left(\sigma, \sigma^{x, y}\right)\right] \\
& =\sum_{\sigma} \mu_{\text {Lie }}(\sigma) \sum_{x, y \in \Lambda} C_{\text {Lie }}\left(\sigma, \sigma^{x, y}\right) F_{\text {Lie }}\left(\sigma, \sigma^{x, y}\right),
\end{aligned}
$$

where $\mu_{\text {Lie }}$ is the corresponding stationary distribution of the Lie scheme, $C_{\text {Lie }}=$ $\left[L_{1}, L_{2}\right]$ and $F_{\text {Lie }}$ depends only on the transition rates. If we consider a state $\sigma$ and a lattice site $x, \sigma^{x}$ corresponds to the resulting state after a spin-flip at that lattice site and $\sigma^{x, y}$ denotes successive spin-flips at $x$ and $y$. Note that $A_{\text {Lie }}$ in Equation (27) seemingly depends on all lattice positions $x, y$. This is also the case for $D_{\text {Lie }}$ and the corresponding coefficients for the Strang splitting (see Appendix B). However, an important property of the commutator in Lemma 1 can be used to simplify the situation and is further explained in Remark 2 .

Remark 2. A key result in [11] was that the commutator is non-zero only for lattice sites on the boundary regions (see Figure 1). This has two major implications:

1. The sums over the lattice $\Lambda$ in the highest order coefficients, $A$ and $D$ (see Equation (24) and (25)), are really sums over the boundary regions, as the commutators for Lie and Strang are non-zero only along the boundary [11, Lemma 5.15].

2. We can compute the scaling of the highest order coefficients, A and D, with the system size.

Due to Remark 2, we can estimate the EPR in a manner that does not depend on the system size by normalizing by the appropriate scaling. For instance, for the adsorption/desorption system on an $N \times N$ lattice, since the boundary scales as $O(N)$, and because the commutator is non-zero only at the boundaries between sub-lattices, that is, $C\left(\sigma, \sigma^{x, y}\right)=0$ if $x, y$ are not in the boundaries of different sub-lattices, the coefficient $A_{\text {Lie }}$ in (27) scales like $O(N)$ too. Specifically for the Lie splitting, the per-particle highest order coefficient of the RER (appearing in Equation (24) as " $A$ ") would be $A / N$. We do this for all estimates in this work, i.e., they are per-lattice-size estimates. Note that the linear scaling is a property of systems that change a single lattice site per jump, such as the adsorption/desorption example. Accordingly, other systems can have different scaling for the computation of the highest order coefficients, see for example the diffusion system in Appendix D.

\subsection{Impact of lattice decomposition on reversibility retention}

One of the choices a practitioner has to make when using parallel KMC is the decomposition of the lattice, for example checkerboard versus stripes (see 
Figures 1,2). Selecting the right decomposition can affect the load-balancing of the algorithm as well as the feasibility of the run. For instance, it may be that the size of the lattice is large enough to prohibit even loading the whole system into the memory of a processor. Then, splitting the lattice into blocks, as in Figure 1, can often bypass this issue, whereas splitting into stripes may not be advantageous. In this section, we give an example of a comparison that can be accomplished by using the EPR and its estimates. Other important comparisons could concern more complicated decompositions of the lattice and how those impact the choice of $\Delta t$ for a fixed EPR tolerance.

However, the choice of decomposition also has an effect on the error the splitting method generates per time step, both for bounded time intervals [11] and for long simulations [12]. This error is controlled by the commutator associated with the scheme, and the analysis in [11] shows that a decomposition into stripes results to reduced error due to the smaller size of the boundary region when compared to a block decomposition when blocks and stripes have the same width, see Figures 1 and 2. By approximating the EPR, we can quantify the long-time effect that the change of decomposition has to the reversibility that each scheme retains per time step. To discuss those issues, we simulated an adsorption/desorption process and used the samples to estimate the EPR. For details about the setup of the example see Appendix C, information about the estimators is in Appendix B.

In Figure 4 we can see how sensitive each scheme is to different decompositions of the lattice. In both cases, the schemes have a smaller EPR estimate when using a stripe versus a block decomposition (where the width of the blocks matches the width of the stripes, see Figures 1,2). In fact, the Strang scheme has consistently better performance in controlling the loss of reversibility with respect to $\Delta t$.

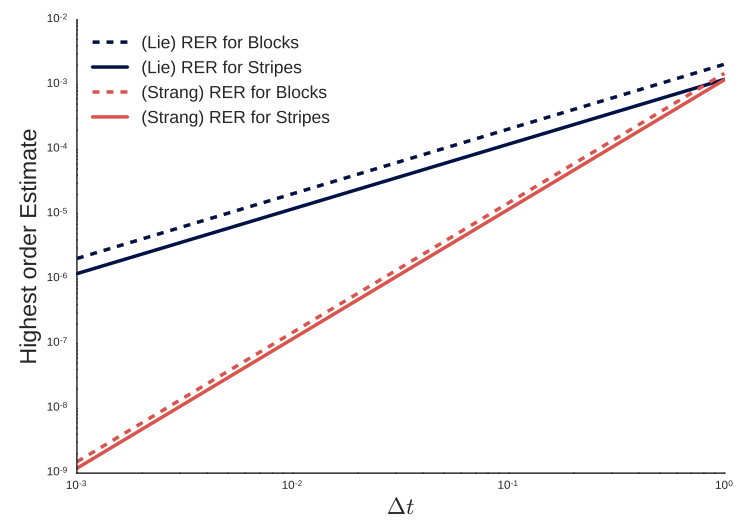

Figure 5: Approximations to the RER of the form $A \cdot \Delta t^{p-1}$ for the same adsorption/desorption system as with Figure 4 for the Lie splitting and Strang splitting. Lie appears to be sensitive to changes in the decomposition of the lattice. 


\section{Derivations and General Theory}

In this section, we present the general theory concerning the asymptotic behavior of the entropy production rate (EPR) of a scheme $Q_{\Delta t}$. The arguments presented here, although mirroring some of the ideas from our previous work [12], also take into account the additional discrepancy term, $I\left(Q_{\Delta t} \mid P_{\Delta t}\right)$. Although we handle only the case that $L$ is split into $L_{1}+L_{2}$, the arguments can also generalize to splittings with more components, e.g. $L_{1}+L_{2}+L_{3}$. In fact, the arguments can readily generalize to schemes that are not splittings, as long as there is an expression for the error like the one in Lemma 1. Nevertheless, we will continue to consider splitting methods in this section.

Remark 3. An implicit assumption in the parallel schemes used in Section 4 was that the splitting of the generator $L$ into $L_{1}+L_{2}$ was such that if $q\left(\sigma, \sigma^{\prime}\right)=0$ for some pair of states $\left(\sigma, \sigma^{\prime}\right)$, then $q_{1}\left(\sigma, \sigma^{\prime}\right)=q_{2}\left(\sigma, \sigma^{\prime}\right)=0$. This is imposed by the domain decomposition of the lattice and we also assume this throughout for any splitting of L, although the methodology can be extended to other splittings too.

\subsection{Decomposition of the Entropy Production Rate}

To better understand the Entropy Production Rate, we shall first decompose it into two pieces, the relative entropy rate, Equation (22), and a "discrepancy" term (Equation (23)) that we will denote with $I$.

Theorem 1. Let $\Delta t>0$ and $P_{\Delta t}$ be a transition probability, with stationary distribution $\mu$, that satisfies detailed balance. Then, if $Q_{\Delta t}$ is an approximation coming from a numerical scheme, we have that

$$
\operatorname{EPR}\left(Q_{\Delta t}\right)=H\left(Q_{\Delta t} \mid P_{\Delta t}\right)+I\left(Q_{\Delta t} \mid P_{\Delta t}\right) .
$$

Proof. In Equation (16), we defined entropy production rate corresponding to $Q_{\Delta t}$ as

$$
\operatorname{EPR}\left(Q_{\Delta t}\right)=\frac{1}{\Delta t} \sum_{\sigma, \sigma^{\prime}} \mu_{\Delta t}(\sigma) Q_{\Delta t}\left(\sigma, \sigma^{\prime}\right) \log \left(\frac{Q_{\Delta t}\left(\sigma, \sigma^{\prime}\right)}{Q_{\Delta t}\left(\sigma^{\prime}, \sigma\right)}\right),
$$

We will first introduce the reversible $P_{\Delta t}$ in Equation (30) as

$$
\Delta t \cdot \operatorname{EPR}\left(Q_{\Delta t}\right)=\sum_{\sigma, \sigma^{\prime}, \sigma^{\prime} \neq \sigma} \mu_{\Delta t}(\sigma) Q_{\Delta t}\left(\sigma, \sigma^{\prime}\right) \log \left(\frac{Q_{\Delta t}\left(\sigma, \sigma^{\prime}\right) P_{\Delta t}\left(\sigma, \sigma^{\prime}\right) P_{\Delta t}\left(\sigma^{\prime}, \sigma\right)}{P_{\Delta t}\left(\sigma, \sigma^{\prime}\right) P_{\Delta t}\left(\sigma^{\prime}, \sigma\right) Q_{\Delta t}\left(\sigma^{\prime}, \sigma\right)}\right) .
$$

This allows us to split the logarithm into three pieces.

$$
\begin{aligned}
\Delta t \cdot \operatorname{EPR}\left(Q_{\Delta t}\right)= & \sum_{\sigma, \sigma^{\prime}} \mu_{\Delta t}(\sigma) Q_{\Delta t}\left(\sigma, \sigma^{\prime}\right) \log \left(\frac{Q_{\Delta t}\left(\sigma, \sigma^{\prime}\right)}{P_{\Delta t}\left(\sigma, \sigma^{\prime}\right)}\right) \\
& +\sum_{\sigma, \sigma^{\prime}} \mu_{\Delta t}(\sigma) Q_{\Delta t}\left(\sigma, \sigma^{\prime}\right) \log \left(\frac{P_{\Delta t}\left(\sigma, \sigma^{\prime}\right)}{P_{\Delta t}\left(\sigma^{\prime}, \sigma\right)}\right) \\
& +\sum_{\sigma, \sigma^{\prime}} \mu_{\Delta t}(\sigma) Q_{\Delta t}\left(\sigma, \sigma^{\prime}\right) \log \left(\frac{P_{\Delta t}\left(\sigma^{\prime}, \sigma\right)}{Q_{\Delta t}\left(\sigma^{\prime}, \sigma\right)}\right) .
\end{aligned}
$$


We shall now show that the middle sum is equal to zero. By our assumptions, we know that the pair $\left(P_{\Delta t}, \mu\right)$ satisfies detailed balance, i.e. $\mu\left(\sigma^{\prime}\right) / \mu(\sigma)=$ $P_{\Delta t}\left(\sigma, \sigma^{\prime}\right) / P_{\Delta t}\left(\sigma^{\prime}, \sigma\right)$. Therefore,

$$
\sum_{\sigma, \sigma^{\prime}} \mu_{\Delta t}(\sigma) Q_{\Delta t}\left(\sigma, \sigma^{\prime}\right) \log \left(\frac{P_{\Delta t}\left(\sigma, \sigma^{\prime}\right)}{P_{\Delta t}\left(\sigma^{\prime}, \sigma\right)}\right)=\sum_{\sigma, \sigma^{\prime}} \mu_{\Delta t}(\sigma) Q_{\Delta t}\left(\sigma, \sigma^{\prime}\right)\left[\log \left(\mu\left(\sigma^{\prime}\right)\right)-\log (\mu(\sigma))\right]
$$

Looking at each sum in Equation (32) separately and using that $\mu_{\Delta t}\left(\sigma^{\prime}\right)=$ $\sum_{\sigma} \mu_{\Delta t}(\sigma) Q_{\Delta t}\left(\sigma, \sigma^{\prime}\right)$, we have

$$
\begin{aligned}
\sum_{\sigma} \sum_{\sigma^{\prime}} \mu_{\Delta t}(\sigma) Q_{\Delta t}\left(\sigma, \sigma^{\prime}\right) \log \left(\mu\left(\sigma^{\prime}\right)\right) & =\sum_{\sigma^{\prime}} \mu_{\Delta t}\left(\sigma^{\prime}\right) \log \left(\mu\left(\sigma^{\prime}\right)\right), \\
\sum_{\sigma} \sum_{\sigma^{\prime}} \mu_{\Delta t}(\sigma) Q_{\Delta t}\left(\sigma, \sigma^{\prime}\right) \log (\mu(\sigma)) & =\sum_{\sigma} \mu_{\Delta t}(\sigma) \log (\mu(\sigma)) .
\end{aligned}
$$

Thus, the right-hand side of Equation (32) is equal to zero and we have,

$$
\begin{aligned}
\Delta t \cdot \operatorname{EPR}\left(Q_{\Delta t}\right)= & \sum_{\sigma, \sigma^{\prime}} \mu_{\Delta t}(\sigma) Q_{\Delta t}\left(\sigma, \sigma^{\prime}\right) \log \left(\frac{Q_{\Delta t}\left(\sigma, \sigma^{\prime}\right)}{P_{\Delta t}\left(\sigma, \sigma^{\prime}\right)}\right) \\
& +\sum_{\sigma, \sigma^{\prime}} \mu_{\Delta t}(\sigma) Q_{\Delta t}\left(\sigma, \sigma^{\prime}\right) \log \left(\frac{P_{\Delta t}\left(\sigma^{\prime}, \sigma\right)}{Q_{\Delta t}\left(\sigma^{\prime}, \sigma\right)}\right) .
\end{aligned}
$$

or

$$
\operatorname{EPR}\left(Q_{\Delta t}\right)=\left(H\left(Q_{\Delta t} \mid P_{\Delta t}\right)+I\left(Q_{\Delta t} \mid P_{\Delta t}\right)\right) / \Delta t
$$

Note that, even though the EPR and the RER are always non-negative, the discrepancy, $I$, is not. If $Q_{\Delta t}$ is reversible, then $\operatorname{EPR}\left(Q_{\Delta t}\right)=0 \Rightarrow H\left(Q_{\Delta t} \mid P_{\Delta t}\right)=$ $-I\left(Q_{\Delta t} \mid P_{\Delta t}\right)$. If, in addition, $Q_{\Delta t} \neq P_{\Delta t}$, then the RER is positive, which implies that $I$ would be negative.

\subsection{Asymptotic Behavior of Entropy Production Rate}

In Theorem 1, we saw that we can express the entropy production rate (EPR) of a scheme as a sum of two different components, the relative entropy rate (Equation (22)) and the discrepancy (Equation (23)). The objective of this section is the study of each component separately via asymptotic expansions with respect to $\Delta t$. Then, at the end of the section we have an asymptotic result for the EPR based on the individual results and Equation (21).

In the derivations that follow, we will often refer to the distances between different states of the state space. A path $\vec{z}$ of length $|\vec{z}|=n$ between states $\sigma, \sigma^{\prime}$ corresponds to a sequence $\vec{z}=\left(z_{0}, \ldots, z_{n}\right)$, with $z_{0}=\sigma, z_{n}=\sigma^{\prime}$, and distinct intermediate states $z_{i}$ such that $\prod_{i=0}^{n} q\left(z_{i}, z_{i+1}\right)>0, q$ being the transition rates 
of the CTMC of interest. The set of all paths between those two states will be denoted by $\operatorname{Path}\left(\sigma \rightarrow \sigma^{\prime}\right)$. We can thus define the distance between two states with respect to a fixed CTMC by the length of the smallest path, $d\left(\sigma, \sigma^{\prime}\right)$. More formally,

$$
d\left(\sigma, \sigma^{\prime}\right):= \begin{cases}\min \left\{|\vec{z}|: \vec{z} \in \operatorname{Path}\left(\sigma \rightarrow \sigma^{\prime}\right)\right\}, & \operatorname{Path}\left(\sigma \rightarrow \sigma^{\prime}\right) \neq \emptyset \\ \infty, & \operatorname{Path}\left(\sigma \rightarrow \sigma^{\prime}\right)=\emptyset\end{cases}
$$

The function $d$ is the geodesic distance and is always calculated with respect to the transition rates $q$ of the exact process, $P_{\Delta t}$. In the time-reversible case, it is simple to show that $d$ is actually a metric of the state space, as it is symmetric and satisfies the triangle inequality. We also define the diameter with respect to $d$ as $\operatorname{diam}(S)=\max _{\left(\sigma, \sigma^{\prime}\right) \in S \times S}\left\{d\left(\sigma, \sigma^{\prime}\right)\right\}$.

We introduced the use of the geodesic distance (33) in Section 8 of [12]. For schemes that satisfy the requirement in Remark 3, the addition of this graphtheoretic perspective can both simplify and generalize the computations. For completeness, we include the result concerning the long-time behavior of the scheme with respect to the RER [12, Theorem 8.6].

Lemma 2. Let $P_{\Delta t}\left(\sigma, \sigma^{\prime}\right)=e^{L \Delta t} \delta_{\sigma^{\prime}}(\sigma)$ and $Q_{\Delta t}\left(\sigma, \sigma^{\prime}\right)$ be an approximation of $P_{\Delta t}$ based on an operator splitting scheme and $\mu_{\Delta t}$ the stationary measure corresponding to $Q_{\Delta t}$. Then, if the scheme is of order $p$, $\operatorname{diam}(S) \geq p$, and $C\left(\sigma, \sigma^{\prime}\right) \neq 0$ for at least one pair $\sigma, \sigma^{\prime} \in S$ such that $d\left(\sigma, \sigma^{\prime}\right)=p$, we have that

$$
H\left(Q_{\Delta t} \mid P_{\Delta t}\right)=O\left(\Delta t^{p-1}\right),
$$

for $\Delta t \leq 1$.

Note that the assumption $\operatorname{diam}(S) \geq p$ is not particularly restrictive for the original Markov process. For example, in lattice systems with adsorption/desorption, diffusion, or other spin-flip mechanisms, consider states that require three jumps of the original Markov process to go from one state to the other. Then $\operatorname{diam}(S) \geq 3$, which is sufficient for the schemes considered here, as the maximum order of the local error is attained by the Strang splitting and is equal to three. Also, checking the existence of a pair $\left(\sigma, \sigma^{\prime}\right)$ for which the commutator $C$ is not zero is just a matter of computation.

Lemma 3. Under the assumptions of Lemma 2, the discrepancy has the same order with the RER. That is,

$$
I\left(Q_{\Delta t} \mid P_{\Delta t}\right)=O\left(\Delta t^{p-1}\right) .
$$

Proof. To show this, we expand $I$ in an asymptotic expansion around $\Delta t$. We demonstrate that the coefficient of the $\Delta t^{p-1}$ term comes from considering the states $\sigma, \sigma^{\prime}$ such that $d\left(\sigma, \sigma^{\prime}\right) \leq p$ and that the dominant order is indeed equal to $p-1$ for small $\Delta t$. We note here that the assumptions on the order, $p$, and the commutator from Lemma 2 are the only assumptions on the operator splitting scheme. 
We defind the discrepancy term in Equation (23) as:

$$
\Delta t \cdot I\left(Q_{\Delta t} \mid P_{\Delta t}\right)=\sum_{\sigma, \sigma^{\prime}} \mu_{\Delta t}(\sigma) Q_{\Delta t}\left(\sigma, \sigma^{\prime}\right) \log \left(\frac{P_{\Delta t}\left(\sigma^{\prime}, \sigma\right)}{Q_{\Delta t}\left(\sigma^{\prime}, \sigma\right)}\right)
$$

Using the atanh representation of the logarithm [12, Equation 5.8] and its expansion, we get that

$\Delta t \cdot I\left(Q_{\Delta t} \mid P_{\Delta t}\right)=\sum_{\sigma, \sigma^{\prime}} \mu_{\Delta t}(\sigma) Q_{\Delta t}\left(\sigma, \sigma^{\prime}\right) \cdot 2 \sum_{k=0}^{\infty} \frac{1}{2 k+1}\left(\frac{P_{\Delta t}\left(\sigma^{\prime}, \sigma\right)-Q_{\Delta t}\left(\sigma^{\prime}, \sigma\right)}{Q_{\Delta t}\left(\sigma^{\prime}, \sigma\right)+P_{\Delta t}\left(\sigma^{\prime}, \sigma\right)}\right)^{2 k+1}$.

In the proof of Lemma 2 (Theorem 5.2 in [12]), we use our knowledge of the asymptotic behavior of $P_{\Delta t} \pm Q_{\Delta t}$ for small $\Delta t[12$, Equations (5.3), (5.4)] to infer the behavior of ratios of those quantities. That is,

$$
\frac{P_{\Delta t}\left(\sigma^{\prime}, \sigma\right)-Q_{\Delta t}\left(\sigma^{\prime}, \sigma\right)}{P_{\Delta t}\left(\sigma^{\prime}, \sigma\right)+Q_{\Delta t}\left(\sigma^{\prime}, \sigma\right)}=\frac{C\left(\sigma^{\prime}, \sigma\right)}{2 Q_{\Delta t}\left(\sigma^{\prime}, \sigma\right)+C\left(\sigma^{\prime}, \sigma\right) \Delta t^{p}} \Delta t^{p}+O\left(\Delta t^{p+1}\right) .
$$

We assume that all $\sigma, \sigma^{\prime}$ satisfy $d\left(\sigma, \sigma^{\prime}\right)=p$, i.e. they are $p$ jumps apart. We define

$$
M\left(\sigma, \sigma^{\prime}\right):=\frac{C\left(\sigma, \sigma^{\prime}\right)}{C\left(\sigma, \sigma^{\prime}\right)+2 L_{Q}^{p}\left(\sigma, \sigma^{\prime}\right) / p !},
$$

where $L_{Q}^{p}$ represents all the terms in the expansion of $Q_{\Delta t}$ that are of order $p$ (see Equation (B.2) in appendix). Then, for $k>0$, we have that

$$
\begin{aligned}
& \sum_{\sigma, \sigma^{\prime}} \mu_{\Delta t}(\sigma) Q_{\Delta t}\left(\sigma, \sigma^{\prime}\right) \cdot 2 \sum_{k=1}^{\infty} \frac{1}{2 k+1}\left(\frac{P_{\Delta t}\left(\sigma^{\prime}, \sigma\right)-Q_{\Delta t}\left(\sigma^{\prime}, \sigma\right)}{P_{\Delta t}\left(\sigma^{\prime}, \sigma\right)+Q_{\Delta t}\left(\sigma^{\prime}, \sigma\right)}\right)^{2 k+1} \\
= & \sum_{\sigma, \sigma^{\prime}} \mu_{\Delta t}(\sigma) L_{Q}^{p}\left(\sigma, \sigma^{\prime}\right) \frac{2 \Delta t^{p}}{p !}\left(\operatorname{atanh}\left(M\left(\sigma^{\prime}, \sigma\right)\right)-M\left(\sigma^{\prime}, \sigma\right)\right)+O\left(\Delta t^{p+1}\right) .
\end{aligned}
$$

Before we continue with the analysis of Equation (37), we look at the term from Equation (34) corresponding to the first term of the series, i.e. $k=0$. Using Equation (35), we get

$$
2 \sum_{\sigma, \sigma^{\prime}} \mu_{\Delta t}(\sigma) Q_{\Delta t}\left(\sigma, \sigma^{\prime}\right) \cdot \frac{C\left(\sigma^{\prime}, \sigma\right)}{2 Q_{\Delta t}\left(\sigma^{\prime}, \sigma\right)+C\left(\sigma^{\prime}, \sigma\right) \Delta t^{p}} \Delta t^{p}+O\left(\Delta t^{p+1}\right) .
$$

We notice that to get terms of order $\Delta t^{p}$ from the sum (38), we need the order of $Q_{\Delta t}\left(\sigma, \sigma^{\prime}\right)$ to be the same as that of $Q_{\Delta t}\left(\sigma^{\prime}, \sigma\right)$. We remind here that the order of the local error is equal to $p$ and that $L^{i}$ is the resulting operator after $i$ compositions of the generator $L$ of the original process. Therefore, if $i<p$, the ratio

$$
\frac{Q_{\Delta t}\left(\sigma, \sigma^{\prime}\right)}{Q_{\Delta t}\left(\sigma^{\prime}, \sigma\right)}=\frac{L^{i}\left(\sigma, \sigma^{\prime}\right) \Delta t^{i}+O\left(\Delta t^{i+1}\right)}{L^{i}\left(\sigma^{\prime}, \sigma\right) \Delta t^{i}+O\left(\Delta t^{i+1}\right)}=\frac{L^{i}\left(\sigma, \sigma^{\prime}\right)}{L^{i}\left(\sigma^{\prime}, \sigma\right)}+o(\Delta t)
$$


is well defined as long as $L^{i}\left(\sigma, \sigma^{\prime}\right) \neq 0$, and that is true because $d\left(\sigma, \sigma^{\prime}\right)=i$ implies that $L^{i}\left(\sigma, \sigma^{\prime}\right)>0$ (see Lemma 6 in Appendix A) and $L^{j}\left(\sigma, \sigma^{\prime}\right)=0$ for $j<i$ [12, Lemma 8.3]. Therefore, the right-hand side of Equation (39) is well-defined for all $\sigma, \sigma^{\prime}$ such that $d\left(\sigma, \sigma^{\prime}\right)=i, i<p$. This finalizes the analysis of the first term of the asymptotic series for $I$, with

$$
\begin{aligned}
& 2 \sum_{\sigma, \sigma^{\prime}} \mu_{\Delta t}(\sigma) Q_{\Delta t}\left(\sigma, \sigma^{\prime}\right) \cdot \frac{C\left(\sigma^{\prime}, \sigma\right)}{2 Q_{\Delta t}\left(\sigma^{\prime}, \sigma\right)+C\left(\sigma^{\prime}, \sigma\right) \Delta t^{p}} \Delta t^{p}+o\left(\Delta t^{p}\right) \\
& =\sum_{\sigma} \mu_{Q}(\sigma) \sum_{i=0}^{p-1} \sum_{\sigma^{\prime} \in S_{i}(\sigma)} \frac{L^{i}\left(\sigma, \sigma^{\prime}\right)}{L^{i}\left(\sigma^{\prime}, \sigma\right)} C\left(\sigma^{\prime}, \sigma\right) \\
& \quad+\sum_{\sigma^{\prime} \in S_{p}(\sigma)} L_{Q}^{p}\left(\sigma, \sigma^{\prime}\right) \frac{2}{p !} M\left(\sigma^{\prime}, \sigma\right) \Delta t^{p}+o\left(\Delta t^{p}\right)
\end{aligned}
$$

Above we use the notation $S_{i}(\sigma)=\left\{\sigma^{\prime}: d\left(\sigma, \sigma^{\prime}\right)=i\right\}$. Now, if we add Equations (37) and (40), the terms that involve $M\left(\sigma^{\prime}, \sigma\right)$ cancel. Thus, we get the following asymptotic expansion for $I$.

$$
\begin{aligned}
I\left(Q_{\Delta t} \mid P_{\Delta t}\right)= & \sum_{\sigma} \mu_{Q}(\sigma) \sum_{i=0}^{p-1} \sum_{\sigma^{\prime} \in S_{i}(\sigma)} \frac{L^{i}\left(\sigma, \sigma^{\prime}\right)}{L^{i}\left(\sigma^{\prime}, \sigma\right)} C\left(\sigma^{\prime}, \sigma\right) \Delta t^{p-1} \\
& +\sum_{\sigma, \sigma^{\prime} \in S_{p}(\sigma)} \mu_{\Delta t}(\sigma) L_{Q}^{p}\left(\sigma, \sigma^{\prime}\right) \frac{2}{p !} \operatorname{atanh}\left(M\left(\sigma^{\prime}, \sigma\right)\right) \Delta t^{p-1} \\
& +o\left(\Delta t^{p-1}\right) .
\end{aligned}
$$

Equation (41) is the basis for our estimation of $I$ for small $\Delta t$, which is used in Section 4. An immediate implication of Theorem 1 and Lemmas 2 and 3 is the next result, which provides the scaling of the EPR with respect to $\Delta t$.

Theorem 2. Let $\Delta t \in(0,1)$. Let $P_{\Delta t}\left(\sigma, \sigma^{\prime}\right)=e^{L \Delta t} \delta_{\sigma^{\prime}}(\sigma)$ and $Q_{\Delta t}\left(\sigma, \sigma^{\prime}\right)$ be an approximation of $P_{\Delta t}$ based on a splitting scheme and $\mu_{\Delta t}$ the stationary measure corresponding to $Q_{\Delta t}$. In addition, let $P_{\Delta t}$ satisfy detailed balance and $\operatorname{diam}(S) \geq p$. Then,

$$
\operatorname{EPR}\left(Q_{\Delta t}\right)=O\left(\Delta t^{p-1}\right) .
$$

Finally, note that $\operatorname{EPR}\left(Q_{\Delta t}\right)=O\left(\Delta t^{p-1}\right)$ implies that the corresponding RER has order $O\left(\Delta t^{p-1}\right)$, or better. In other words, a numerical scheme of high leading order in EPR is also more accurate in sampling from the stationary regime [12]. 


\section{Conclusions}

We introduced the entropy production rate (EPR) as a means to quantify the loss of reversibility for operator splitting schemes applied to Parallel Kinetic Monte Carlo. We showed estimation of the EPR does not require the knowledge of the stationary distribution and depends on the transition probabilities of the scheme. Since the transition probabilities for stochastic particle systems are usually not available, or difficult to explicitly compute, we derived a posteriori estimators of the EPR and connected the parameters of the scheme with a quantitative assessment of the loss of reversibility. We demonstrated this fact with an application to lattice KMC with adsorption/desorption dynamics, which we simulated using SPPARKS [9], and a comparison between two splitting schemes, Lie and Strang. Theory and simulations show that the Strang splitting retains more reversibility per time step compared to Lie and is more stable with respect to changes in the decomposition of the lattice (blocks versus stripes, see Figure 4).

The proposed framework for Parallel KMC, can be applied to more than computational schedule comparison. In essence, the EPR can be used as an information criterion that allows practitioners to judge the fine details of the scheme itself, like the time step and which lattice decompositions retain more reversibility (see Figure 4). The EPR can also be used as a diagnostic observable to assess the reversibility of the scheme used by simulating a system of smaller size than the one of interest. In this way, issues with the scheme can be discovered early on using a much smaller system for diagnostics, different schemes can be compared, and parameters tuned to minimize the loss of reversibility.

Though we only considered operator splitting schemes in the context of parallel lattice $\mathrm{KMC}$, the idea of using the EPR for the quantification of the loss of reversibility can be used on other schemes too, as long as an expression for their local error exists and is computable. For instance, an extension of this work can be used to quantify the loss of reversibility for schemes used for thermostated Molecular Dynamics simulations [29], for example for Langevin dynamics [30].

\section{Acknowledgments}

The work of KG and MAK was partially supported by the Office of Advanced Scientific Computing Research, U.S. Department of Energy, under Contract No. DE-SC0010723. The work of LRB was partially supported by the Office of Advanced Scientific Computing Research, U.S. Department of Energy, under Contract No. DE-SC0010723 and the National Science Foundation under Grant No. DMS 1515172.

\section{References}

[1] Alfred B Bortz, Malvin H Kalos, and Joel L Lebowitz. A new algorithm for Monte Carlo simulation of Ising spin systems. Journal of Computational Physics, 17(1):10-18, 1975. 
[2] Malvin H. Kalos and Paula A. Whitlock. Monte Carlo Methods. WileyInterscience, New York, NY, USA, 1986.

[3] David Landau and Kurt Binder. A Guide to Monte Carlo Simulations in Statistical Physics. Cambridge University Press, New York, NY, USA, 2005 .

[4] Boris D Lubachevsky. Efficient parallel simulations of dynamic Ising spin systems. Journal of Computational Physics, 75(1):103-122, 1988.

[5] Yunsic Shim and Jacques G. Amar. Semirigorous synchronous sublattice algorithm for parallel kinetic Monte Carlo simulations of thin film growth. Phys. Rev. B, 71:125432, Mar 2005.

[6] E. Martnez, J. Marian, M.H. Kalos, and J.M. Perlado. Synchronous parallel kinetic Monte Carlo for continuum diffusion-reaction systems. Journal of Computational Physics, 227(8):3804 - 3823, 2008.

[7] NJ van der Kaap and LJA Koster. Massively parallel kinetic Monte Carlo simulations of charge carrier transport in organic semiconductors. Journal of Computational Physics, 307:321-332, 2016.

[8] Eishin Endo, Yuta Toga, and Munetaka Sasaki. Parallelized stochastic cutoff method for long-range interacting systems. Journal of the Physical Society of Japan, 84(7):074002, 2015.

[9] Steve Plimpton, Corbett Battaile, Mike Chandross, Liz Holm, Aidan Thompson, Veena Tikare, Greg Wagner, E Webb, X Zhou, C Garcia Cardona, et al. Crossing the mesoscale no-mans land via parallel kinetic Monte Carlo. Sandia Report SAND2009-6226, 2009.

[10] Giorgos Arampatzis, Markos A. Katsoulakis, Petr Plecháč, Michela Taufer, and Lifan Xu. Hierarchical fractional-step approximations and parallel kinetic Monte Carlo algorithms. J. Comput. Phys., 231(23):7795-7814, October 2012.

[11] Giorgos Arampatzis, Markos A. Katsoulakis, and Petr Plecháč. Parallelization, processor communication and error analysis in lattice kinetic Monte Carlo. SIAM Journal on Numerical Analysis, 52(3):1156-1182, 2014.

[12] K. Gourgoulias, M. A. Katsoulakis, and L. Rey-Bellet. Information metrics for long-time errors in splitting schemes for stochastic dynamics and parallel kMC. pre-print. arXiv:1511.08240 [math.NA].

[13] Jerome P. Nilmeier and Jaime Marian. A rigorous sequential update strategy for parallel kinetic Monte Carlo simulation. Computer Physics Communications, 185(10):2479 - 2486, 2014. 
[14] Markos Katsoulakis, Yannis Pantazis, and Luc Rey-Bellet. Measuring the irreversibility of numerical schemes for reversible stochastic differential equations. ESAIM: Mathematical Modelling and Numerical Analysis, 48:1351-1379, 92014.

[15] Christian Maes. The fluctuation theorem as a Gibbs property. Journal of statistical physics, 95(1-2):367-392, 1999.

[16] Christian Maes, Frank Redig, and Annelies Van Moffaert. On the definition of entropy production, via examples. Journal of Mathematical Physics, 41(3):1528-1554, 2000.

[17] Joel L. Lebowitz and Herbert Spohn. A Gallavotti-Cohen-type symmetry in the large deviation functional for stochastic dynamics. Journal of Statistical Physics, 95(1):333-365.

[18] Jorge Kurchan. Fluctuation theorem for stochastic dynamics. Journal of Physics A: Mathematical and General, 31(16):3719, 1998.

[19] Giovanni Gallavotti and EGD Cohen. Dynamical ensembles in stationary states. Journal of Statistical Physics, 80(5-6):931-970, 1995.

[20] Yannis Pantazis and Markos A. Katsoulakis. A relative entropy rate method for path space sensitivity analysis of stationary complex stochastic dynamics. The Journal of Chemical Physics, 138(5), 2013.

[21] Evangelia Kalligiannaki, Markos A. Katsoulakis, and Petr Plecháč. Spatial two-level interacting particle simulations and information theory-based error quantification. SIAM Journal on Scientific Computing, 36(2):A634A667, 2014.

[22] H. F. Trotter. On the product of semi-groups of operators. Proceedings of the American Mathematical Society, 10(4):pp. 545-551, 1959.

[23] Eskil Hansen and Alexander Ostermann. Exponential splitting for unbounded operators. Mathematics of computation, 78(267):1485-1496, 2009.

[24] Tobias Jahnke and Derya Altntan. Efficient simulation of discrete stochastic reaction systems with a splitting method. BIT Numerical Mathematics, 50(4):797-822, 2010.

[25] Frank P Kelly. Reversibility and stochastic networks. Cambridge University Press, 2011.

[26] Thomas M. Cover and Joy A. Thomas. Elements of Information Theory. Wiley-Interscience, New York, NY, USA, 1991.

[27] Hirotogu Akaike. Information theory and an extension of the maximum likelihood principle. In Emanuel Parzen, Kunio Tanabe, and Genshiro Kitagawa, editors, Selected Papers of Hirotugu Akaike, Springer Series in Statistics, pages 199-213. Springer New York, 1998. 
[28] Hirotugu Akaike. A new look at the Bayes procedure. Biometrika, 65(1):5359, 1978.

[29] Ben Leimkuhler and Charles Matthews. Molecular Dynamics: with deterministic and stochastic numerical methods, volume 39. Springer, 2015.

[30] Benedict Leimkuhler, Charles Matthews, and Gabriel Stoltz. The computation of averages from equilibrium and nonequilibrium Langevin molecular dynamics. IMA Journal of Numerical Analysis, 2015.

\section{Appendix A. Supporting Results}

In this section, we provide proofs for supporting results in the main manuscript.

Let $X_{n}$ be a Markov process with $P$ the Markov transition kernel and $\mu$ the corresponding stationary distribution. Also,

$$
p\left(\sigma_{0}, \ldots, \sigma_{m}\right)=p\left(X_{0}=\sigma_{0}, \ldots, X_{m}=\sigma_{0}\right),
$$

$\sigma_{i}$ being states of the process from a state space $S$. We also use the notation $\sigma_{0: m}$ for the sequence of states $\sigma_{0}, \ldots, \sigma_{m}$. In some cases those states will have to be distinct, and this will be mentioned separately when is needed.

Appendix A.1. Connection of the Entropy Production with the Entropy Production Rate

In the main text (see Equations (14), (15)), we sketched a proof for the connection between entropy production (EP) for paths of length $m$,

$$
\operatorname{EP}(P)=\sum_{\sigma_{0: m}} p\left(\sigma_{0: m}\right) \log \left(\frac{p\left(\sigma_{0: m}\right)}{p\left(\sigma_{m: 0}\right)}\right)
$$

and entropy production per unit time (or entropy production rate (EPR)),

$$
\operatorname{EPR}(P)=\sum_{\sigma, \sigma^{\prime}} \mu(\sigma) P\left(\sigma, \sigma^{\prime}\right) \log \left(\frac{P\left(\sigma, \sigma^{\prime}\right)}{P\left(\sigma^{\prime}, \sigma\right)}\right)
$$

Lemma 4. let $m \in \mathbb{N}$ and let $P$ be a Markov transition probability kernel, with $\mu$ being the stationary distribution that corresponds to $P$. Then,

$$
\operatorname{EP}(P)=m \cdot \sum_{\sigma_{0}, \sigma_{1}} \mu\left(\sigma_{0}\right) P\left(\sigma_{0}, \sigma_{1}\right) \log \left(\frac{P\left(\sigma_{0}, \sigma_{1}\right)}{P\left(\sigma_{1}, \sigma_{0}\right)}\right)=m \cdot \operatorname{EPR}(P) .
$$

Proof. By the Markov property, we can express $p\left(\sigma_{0: m}\right), p\left(\sigma_{m: 0}\right)$ with respect to $P, \mu$ :

$$
\begin{aligned}
& p\left(\sigma_{0: m}\right)=\mu\left(\sigma_{0}\right) P\left(\sigma_{0}, \sigma_{1}\right) \cdots P\left(\sigma_{m-1}, \sigma_{m}\right), \\
& p\left(\sigma_{m: 0}\right)=\mu\left(\sigma_{m}\right) P\left(\sigma_{m}, \sigma_{m-1}\right) \cdots P\left(\sigma_{1}, \sigma_{0}\right) .
\end{aligned}
$$


Substituting those in the definition of the EP in Equation (A.1), we get

$$
\begin{aligned}
& \sum_{\sigma_{0: m}} \mu\left(\sigma_{0}\right) P\left(\sigma_{0}, \sigma_{1}\right) \cdots P\left(\sigma_{m-1}, \sigma_{m}\right) \log \left(\frac{\mu\left(\sigma_{0}\right) P\left(\sigma_{0}, \sigma_{1}\right) \cdots P\left(\sigma_{m-1}, \sigma_{m}\right)}{\mu\left(\sigma_{m}\right) P\left(\sigma_{m}, \sigma_{m-1}\right) \cdots P\left(\sigma_{1}, \sigma_{0}\right)}\right) \\
& =\sum_{\sigma_{0: m}} \mu\left(\sigma_{0}\right) P\left(\sigma_{0}, \sigma_{1}\right) \cdots P\left(\sigma_{m-1}, \sigma_{m}\right) \log \left(\frac{\mu\left(\sigma_{0}\right)}{\mu\left(\sigma_{m}\right)}\right) \\
& +\sum_{\sigma_{0: m}} \mu\left(\sigma_{0}\right) P\left(\sigma_{0}, \sigma_{1}\right) \cdots P\left(\sigma_{m-1}, \sigma_{m}\right) \sum_{k=1}^{m} \log \left(\frac{P\left(\sigma_{k-1}, \sigma_{k}\right)}{P\left(\sigma_{k}, \sigma_{k-1}\right)}\right) .
\end{aligned}
$$

First, we shall show that Equation (A.5) is equal to zero. We can write it as

$$
\begin{aligned}
& \sum_{\sigma_{0: m}} \mu\left(\sigma_{0}\right) \log \left(\mu\left(\sigma_{0}\right)\right) P\left(\sigma_{0}, \sigma_{1}\right) \cdots P\left(\sigma_{m-1}, \sigma_{m}\right) \\
& -\sum_{\sigma_{0: m}} \mu\left(\sigma_{0}\right) P\left(\sigma_{0}, \sigma_{1}\right) \cdots P\left(\sigma_{m-1}, \sigma_{m}\right) \log \left(\mu\left(\sigma_{m}\right)\right) .
\end{aligned}
$$

Now, for the first sum, we can repeatedly use that

$$
\sum_{\sigma^{\prime}} P\left(\sigma, \sigma^{\prime}\right)=1
$$

for all states $\sigma$, which results to Equation (A.7) being reduced to

$$
\sum_{\sigma_{0}} \mu\left(\sigma_{0}\right) \log \left(\mu\left(\sigma_{0}\right)\right)
$$

For the part in (A.8), since $\mu$ is the stationary distribution associated with $P$, we have that for any state $\sigma^{\prime}$,

$$
\mu\left(\sigma^{\prime}\right)=\sum_{\sigma} \mu(\sigma) P\left(\sigma, \sigma^{\prime}\right)
$$

Using the property in (A.10) repeatedly on Equation (A.8), we get that it is equal to (A.7), which gives the equality of the first sum in the right-hand side of Equation (A.5) to zero. Next, we need to account for (A.6), which we write as

$$
\sum_{k=1}^{m} \sum_{\sigma_{0: m}} \mu\left(\sigma_{0}\right) P\left(\sigma_{0}, \sigma_{1}\right) \cdots P\left(\sigma_{m-1}, \sigma_{m}\right) \log \left(\frac{P\left(\sigma_{k-1}, \sigma_{k}\right)}{P\left(\sigma_{k}, \sigma_{k-1}\right)}\right) .
$$

For $k=1$, and by using property (A.9), we get

$$
\sum_{\sigma_{0: 1}} \mu\left(\sigma_{0}\right) P\left(\sigma_{0}, \sigma_{1}\right) \log \left(\frac{P\left(\sigma_{0}, \sigma_{1}\right)}{P\left(\sigma_{1}, \sigma_{0}\right)}\right) .
$$

For any other $k$ in Equation (A.11), we can use Equation (A.10) to show that all terms are equal to (A.12). Since we have $m$ of those, this proves the result.

The technique with which we showed that the term in (A.5) is equal to zero is a generalization of the one we used in the proof of Theorem 1 in the main text (see from Equation (31) in the main text and below). 


\section{Appendix A.2. Connectivity and Markov generators}

We remind here that $L$ is a generator of a Markov process $X_{n}, L^{k}$ represents the result of $k$ compositions of $L . d$ is the geodesic distance between states, defined with respect to the transition rates of the exact Markov process with transition probabilities $P_{\Delta t}$ :

$$
d\left(\sigma, \sigma^{\prime}\right):= \begin{cases}\min \left\{|\vec{z}|: \vec{z} \in \operatorname{Path}\left(\sigma \rightarrow \sigma^{\prime}\right)\right\}, & \operatorname{Path}\left(\sigma \rightarrow \sigma^{\prime}\right) \neq \emptyset, \\ \infty, & \operatorname{Path}\left(\sigma \rightarrow \sigma^{\prime}\right)=\emptyset .\end{cases}
$$

In (A.13), $|\vec{z}|$ is the length of a path from $\sigma$ to $\sigma^{\prime}$ and $\operatorname{Path}\left(\sigma \rightarrow \sigma^{\prime}\right)$ corresponds to the set of all such possible paths connecting $\sigma$ and $\sigma^{\prime}$.

In the proof of Lemma 3 in the main text we used that if we have two states $\sigma, \sigma^{\prime}$ with $d\left(\sigma, \sigma^{\prime}\right)=k$, then $L^{k}\left(\sigma, \sigma^{\prime}\right)>0$. This is a consequence of a specific representation that $L^{k}\left(\sigma, \sigma^{\prime}\right)$ has when the states $\sigma$ and $\sigma^{\prime}$ are $k$ steps apart.

Lemma 5. Let $\sigma, \sigma^{\prime} \in S$ and let $L$ be the generator of the Markov process. Then

$$
d\left(\sigma, \sigma^{\prime}\right)=k \Rightarrow L^{k}\left(\sigma, \sigma^{\prime}\right)=\sum_{z_{1: k-1}} q\left(\sigma, z_{1}\right) \ldots q\left(z_{k-1}, \sigma^{\prime}\right) .
$$

Note the notation $z_{1: n-1}=\left(z_{1}, \ldots, z_{n-1}\right)$ for a path of states of length $n-1$. Here we assume that $\sigma, z_{1}, \ldots, z_{n-1}, \sigma^{\prime}$ are distinct states, so that the path from $\sigma$ to $\sigma^{\prime}$ is of length $n$.

Proof. The result is immediate for $k=0$ or $k=1$, as $L^{0}(\sigma, \sigma)=\delta_{\sigma}(\sigma)=1$ and $L\left(\sigma, \sigma^{\prime}\right)=q\left(\sigma, \sigma^{\prime}\right)$, since there is only one path between $\sigma$ and $\sigma^{\prime}$. Let us assume that this fact holds for $k=n$. That is, for states such that $d\left(\sigma, \sigma^{\prime}\right)=n$,

$$
L^{n}\left(\sigma, \sigma^{\prime}\right)=\sum_{z_{1: n-1}} q\left(\sigma, z_{1}\right) \ldots q\left(z_{n-1}, \sigma^{\prime}\right) .
$$

Note that in Equation (A.14), we have a sum that contains all paths of length $n$ connecting $\sigma$ to $\sigma^{\prime}$. As the states in the sum are distinct, the product $q\left(\sigma, z_{1}\right) \ldots q\left(z_{n-1}, \sigma^{\prime}\right)$ is always non-negative. In fact, an implication of representation (A.14) for $L^{n}\left(\sigma, \sigma^{\prime}\right)$ is that $L^{n}$ is positive when the states $\sigma$ and $\sigma^{\prime}$ are $n$ steps apart. We demonstrate this now as it will be useful for the rest of the proof. Consider a path of states of length $n$ from $b_{0}=\sigma$ to $b_{n}=\sigma^{\prime}$, $\left(b_{0}, b_{1}, \ldots, b_{n-1}, b_{n}\right)$, where the $z_{i}$ are all distinct states. Then, as that sequence of states is a path, we have $q\left(b_{i}, b_{i+1}\right)>0$ for $i=0, \ldots, n-1$. However, this path is also contained in the sum in Equation (A.14). Therefore, we have

$$
L^{n}\left(\sigma, \sigma^{\prime}\right)=\sum_{z_{1: n-1}} q\left(\sigma, z_{1}\right) \ldots q\left(z_{n-1}, \sigma^{\prime}\right) \geq q\left(\sigma, b_{1}\right) \ldots q\left(b_{n-1}, \sigma^{\prime}\right)>0 .
$$


We will now show the result for $d\left(\sigma, \sigma^{\prime}\right)=n+1$. Since $L^{n+1}$ is $L$ after $n+1$ compositions, we can write

$$
L^{n+1}\left(\sigma, \sigma^{\prime}\right)=L\left[L^{n}\left[\delta_{\sigma^{\prime}}\right]\right](\sigma) .
$$

Then, by the definition of the generator $L$,

$$
\begin{aligned}
L\left[L^{n}\left[\delta_{\sigma^{\prime}}\right]\right](\sigma) & =\sum_{z} q(\sigma, z)\left(L^{n}\left[\delta_{\sigma^{\prime}}\right](z)-L^{n}\left[\delta_{\sigma^{\prime}}\right](\sigma)\right) \\
& =\sum_{z} q(\sigma, z) L^{n}\left[\delta_{\sigma^{\prime}}\right](z)
\end{aligned}
$$

In (A.16), we used that $d\left(\sigma, \sigma^{\prime}\right)=n+1 \Rightarrow L^{n}\left[\delta_{\sigma^{\prime}}\right](\sigma)=0$. This is true by the induction hypothesis we made in Equation (A.14). If $q(\sigma, z)=0$, the corresponding terms are also zero, so let $z$ be a state such that $q(\sigma, z)>0$. As we argued above, due to the representation in (A.14) $L^{n}\left(z, \sigma^{\prime}\right)>0$. Thus, we will now show that

$$
n \leq d\left(z, \sigma^{\prime}\right) \leq n+2 .
$$

For the upper bound, we apply the triangle inequality. To get the lower, if $d(\sigma, z)=1$ and $d\left(z, \sigma^{\prime}\right)$ is lower or equal to $n-1$, then by following the path $\sigma \rightarrow z \rightarrow \sigma^{\prime}$, we get a new path between $\sigma$ and $\sigma^{\prime}$ with at most $n$ steps. This contradicts that $d\left(\sigma, \sigma^{\prime}\right)$ is the minimum number of steps to get from $\sigma$ to $\sigma^{\prime}$, as we have already assumed that $d\left(\sigma, \sigma^{\prime}\right)=n+1$.

Now, since $d\left(\sigma, \sigma^{\prime}\right)>n \Rightarrow L^{n}\left[\delta_{\sigma^{\prime}}\right](\sigma)=0$, we get that only the pairs of states $\left(z, \sigma^{\prime}\right)$ such that $d\left(z, \sigma^{\prime}\right)=n$ lead to potential non-zero terms for the sum in Equation (A.16). Therefore, if we assume $d\left(z, \sigma^{\prime}\right)=n$, and by using the induction step in Equation (A.16), we have

$$
L^{n+1}\left(\sigma, \sigma^{\prime}\right)=L\left[L^{n}\left[\delta_{\sigma^{\prime}}\right]\right](\sigma)=\sum_{z, z_{1: n-1}} q(\sigma, z) q\left(\sigma, z_{1}\right) \ldots q\left(z_{n-1}, \sigma^{\prime}\right) .
$$

While proving Lemma 5, we also demonstrated that compositions of the generators are always positive on certain pairs of states.

Lemma 6. Let $\sigma, \sigma^{\prime}$ be states such that $d\left(\sigma, \sigma^{\prime}\right)=k$. Then $L^{k}\left(\sigma, \sigma^{\prime}\right)>0$.

Proof. This is a corollary of Lemma 5.

Appendix B. Highest-order coefficients for Lie and Strang operator splitting schemes

Let $L$ be a bounded operator, which allows us to represent the semigroup $e^{L t}$ via a power series expansion. We shall use the notation $L\left(\sigma, \sigma^{\prime}\right):=L\left[\delta_{\sigma}^{\prime}\right](\sigma)$, with which we have

$$
P_{t}\left(\sigma, \sigma^{\prime}\right)=e^{L t} \delta_{\sigma^{\prime}}(\sigma)=\sum_{k=0}^{\infty} \frac{L^{k}\left(\sigma, \sigma^{\prime}\right)}{k !} t^{k}
$$


We assume that we can write an expansion for $Q_{\Delta t}$ too by representing each semigroup in Equation (3) and Equation (4) by its series and then multiplying out. By this process, we get

$$
Q_{\Delta t}\left(\sigma, \sigma^{\prime}\right)=\sum_{k=0}^{\infty} \frac{L_{Q}^{k}\left(\sigma, \sigma^{\prime}\right)}{k !} \Delta t^{k},
$$

where $L_{Q}^{k}$ represents the terms of order $k$ in the expansion of $Q_{\Delta t}$. For example, for the Lie splitting, $L_{\mathrm{Lie}}^{0}=I, L_{\mathrm{Lie}}^{1}=L, L_{\text {Lie }}^{2}=\left(L_{1}^{2}+L_{2}^{2}+2 L_{1} L_{2}\right)$. In general, the exact form of $L_{Q}^{k}$ can be computed by using the $\mathrm{BCH}$ formula. This notation is picked for clarity and does not imply that $L_{Q}$ is a generator of a Markov process. As such, $L_{Q}^{k}$ does not equal $k$ compositions of $L_{Q}$, except if $k<p, p$ being the order of the local error for the operator splitting scheme.

Lemma 7 and Lemma 8 demonstrate the form of the highest-order coefficients of the RER and the discrepancy for the Lie and Strang schemes in the case that $d\left(\sigma, \sigma^{\prime}\right)=1$ implies $\sigma^{\prime}=\sigma^{x}$ for some $x$ in the lattice. This includes the adsorption/desorption systems, an example of which was demonstrated in Section 4.

Lemma 7. Under the assumptions of Lemma 2, if $A_{\mathrm{Lie}}\left(A_{\mathrm{Str}}\right)$ is the highest order coefficient of the RER for the Lie (Strang) splitting, then

$$
\begin{aligned}
& A_{\text {Lie }}=\mathbb{E}_{\mu_{\text {Lie }}(\sigma)}\left[\sum_{x, y \in \Lambda} F_{\text {Lie }}\left(\sigma, \sigma^{x, y}\right)\right]=\sum_{\sigma} \mu_{\text {Lie }}(\sigma) \sum_{x, y \in \Lambda} F_{\text {Lie }}\left(\sigma, \sigma^{x, y}\right), \\
& F_{\text {Lie }}\left(\sigma, \sigma^{\prime}\right):=C_{\text {Lie }}\left(\sigma, \sigma^{\prime}\right) M_{\text {Lie }}\left(\sigma, \sigma^{\prime}\right)-2 L_{\text {Lie }}^{2}\left[\delta_{\sigma^{\prime}}\right](\sigma)\left(\operatorname{arctanh}\left(M_{\text {Lie }}\left(\sigma, \sigma^{\prime}\right)\right)-M_{\text {Lie }}\left(\sigma, \sigma^{\prime}\right)\right), \\
& M_{\text {Lie }}\left(\sigma, \sigma^{\prime}\right):=C_{\text {Lie }}\left(\sigma, \sigma^{\prime}\right) /\left(L_{\text {Lie }}^{2}\left[\delta_{\sigma^{\prime}}(\sigma)\right]+C_{\text {Lie }}\left(\sigma, \sigma^{\prime}\right)\right)
\end{aligned}
$$

$C_{\mathrm{Li}}$ stands for the commutator of the Lie scheme, $C_{\mathrm{L}}$

For the Strang splitting,

$$
\begin{aligned}
& A_{\mathrm{Str}}=\mathbb{E}_{\mu_{\mathrm{Str}}(\sigma)}\left[\sum_{x, y, z \in \Lambda} F_{\mathrm{Str}}\left(\sigma, \sigma^{x, y, z}\right)\right]=\sum_{\sigma} \mu_{\mathrm{Str}}(\sigma) \sum_{x, y, z \in \Lambda} F_{\mathrm{Str}}\left(\sigma, \sigma^{x, y, z}\right), \\
& F_{\mathrm{Str}}\left(\sigma, \sigma^{\prime}\right):=C_{\mathrm{Str}}\left(\sigma, \sigma^{\prime}\right) M_{\mathrm{Str}}\left(\sigma, \sigma^{\prime}\right)-2 L_{\mathrm{Str}}^{3}\left[\delta_{\sigma^{\prime}}\right](\sigma)\left(\operatorname{arctanh}\left(M_{\mathrm{Str}}\left(\sigma, \sigma^{\prime}\right)\right)-M_{\mathrm{Str}}\left(\sigma, \sigma^{\prime}\right)\right), \\
& M_{\mathrm{Str}}\left(\sigma, \sigma^{\prime}\right):=C_{\mathrm{Str}}\left(\sigma, \sigma^{\prime}\right) /\left(L_{\mathrm{Str}}^{3}\left[\delta_{\sigma^{\prime}}\right](\sigma)+C_{\mathrm{Str}}\left(\sigma, \sigma^{\prime}\right)\right) .
\end{aligned}
$$

Proof. See proof of Theorem 5.2 in [12].

Similarly, from the proof of Lemma 3, Section 5, and specifically Equation (38), we can write down the highest-order coefficient for the discrepancy. 
Lemma 8. Under the assumptions of Theorem 3, if $D_{\mathrm{Lie}}\left(D_{\mathrm{Str}}\right)$ is the highest order coefficient of I for the Lie (Strang) splitting, then

$$
\begin{aligned}
D_{\mathrm{Lie}}= & \sum_{\sigma} \mu_{\text {Lie }}(\sigma) \sum_{x \in \Lambda} \frac{q\left(\sigma, \sigma^{x}\right)}{q\left(\sigma^{x}, \sigma\right)} C_{\mathrm{Lie}}\left(\sigma^{x}, \sigma\right) \\
& +\sum_{x, y \in \Lambda} \mu_{\mathrm{Lie}}(\sigma) L_{\mathrm{Lie}}^{2}\left(\sigma, \sigma^{x, y}\right) \operatorname{atanh}\left(M_{\mathrm{Lie}}\left(\sigma^{x, y}, \sigma\right)\right) .
\end{aligned}
$$

and

$$
\begin{aligned}
D_{\mathrm{Str}}= & \sum_{\sigma} \mu_{\mathrm{Str}}(\sigma)\left(\sum_{x \in \Lambda} \frac{q\left(\sigma, \sigma^{x}\right)}{q\left(\sigma^{x}, \sigma\right)} C_{\mathrm{Str}}\left(\sigma^{x}, \sigma\right)+\sum_{x, y \in \Lambda} \frac{L^{2}\left(\sigma, \sigma^{x, y}\right)}{L^{2}\left(\sigma^{x, y}, \sigma\right)} C_{\mathrm{Str}}\left(\sigma^{x, y}, \sigma\right)\right. \\
& \left.+\sum_{x, y, z \in \Lambda} L_{\mathrm{Str}}^{3}\left(\sigma, \sigma^{x, y, z}\right) \frac{1}{3} \operatorname{atanh}\left(M_{\mathrm{Str}}\left(\sigma^{x, y, z}, \sigma\right)\right)\right) .
\end{aligned}
$$

Note that the sums over the lattice sites $x, y$ are in fact sums over the boundary region due to the properties of the commutator, see discussion around Remark 2. Although the coefficients have forms that depend on the transition rates and are given here explicitly, their estimation can be complicated for more complex systems, see diffusion example in Appendix D.

\section{Appendix C. Adsorption/Desorption Example}

Here we include the setup for the adsorption/desorption example we simulated with the help of SPPARKS.

Let $\Lambda \subset \mathbb{Z}^{2}$ be a bounded, two-dimensional integer lattice with dimensions $N \times N$. To every lattice site $x$ corresponds a spin variable $\sigma(x), \sigma(x) \in \Sigma=$ $\{0,1\}$, where $\sigma(x)=0$ denotes that site $x$ is empty and $\sigma(x)=1$ that the site is occupied by some particle. The transition rates will correspond to single spin-flip Arrhenius dynamics. If we fix a state $\sigma \in S$ and a lattice site $x \in \Lambda$, then the transition rates $q$ are defined by

$$
\begin{aligned}
q\left(\sigma, \sigma^{x}\right) & =q(x, \sigma)=c_{1}(1-\sigma(x))+c_{2} \sigma(x) e^{-\beta U(x)}, \\
U(x, \sigma) & =J_{0} \sum_{y \in \Omega_{x}} \sigma(y)+h .
\end{aligned}
$$

The constants, $c_{1}, c_{2}, \beta, J_{0}, h$, can be tuned to generate different dynamics. $\sigma^{x}$ is the resulting state after starting with $\sigma$ and changing $\sigma(x)$ to $1-\sigma(x)$. $\Omega_{x}$ represents the set of lattice sites that are neighbors of $x$. For this model, $\Omega_{x}$ will just be the nearest neighbors of $x$, like in Figure 1. The single spin-flip process, defined by the transition rates in (C.1), satisfies detailed balance and can be simulated exactly via Kinetic Monte Carlo. 
To produce Figures 3, 4, and 5, we simulated an adsorption-desorption system with the Lie and Strang schemes in SPPARKS, with rate constants $c_{1}=c_{2}=1, \beta=2, J_{0}=0.3$, and $h=0.9$, and starting from various configurations of the lattice. The particular plots are for the case that in every lattice site sits a particle. In order to estimate the EPR by using the expressions in Lemmas 7 and 8 , we selected a splitting time-step $\Delta t=0.001$ and simulated the process in time for $T=100, N=100$, while simultaneously tracking the mean coverage of the lattice (to assess equilibration of the system). Then the approximation to the EPR for the $\Delta t$ considered is given by $(A+D) \Delta t^{p-1}$.

\section{Appendix D. Estimators of the EPR for a Diffusion Process}

To show how the calculation of the estimators would change under a different model, we shall now demonstrate the case of a diffusion process. Let us assume that it is modeled by the set of transition rates

$$
q(x, y, \sigma)=p(x, y) \sigma(x)(1-\sigma(y)), x, y \in \Lambda
$$

for some state $\sigma$. At each time step, the system can swap the values between two lattice sites, $x, y . p(x, y)$ corresponds to some decaying potential that captures the distance a particle can travel. For instance, for nearest neighbor jumps, we would have $p(x, y)=1 / 4$ if $|x-y|=1$, and $p(x, y)=0$ otherwise. Note that the transition rates $q$ are zero if the origin site $x$ is empty or if the target site $y$ is occupied.

We focus on the case of computing the discrepancy term, $I$, for the Lie splitting with a splitting of the generator $L$ into $L_{1}+L_{2}$. Nevertheless, this example will also be instructive for the case of the relative entropy rate and other splittings. Theorems 2 and 3 make no assumption on the underlying model. They do however use the notion of distance between states that the transition rates define (see discussion at the beginning of Section 5). For this model, two states $\sigma, \sigma^{\prime}$, are one jump apart if there exist distinct lattice sites $x, y$ such that $\sigma^{\prime}=\sigma^{x, y}$, and two jumps apart if there exist distinct $x, y, z, w$ such that $\sigma^{\prime}=\sigma^{x, y, z, w}$. The notation $\sigma^{x, y, z, w}$ denotes the resulting state after starting with a state $\sigma$ and carrying out spin-flips at the lattice locations $x, y, z, w$.

After computing the corresponding commutator, $C_{\text {Lie }}\left(\sigma, \sigma^{\prime}\right)=\left[L_{1}, L_{2}\right] \delta_{\sigma^{\prime}}(\sigma)$, and $L_{\text {Lie }}^{2}$, we can write the exact formula for the highest order coefficient for the Lie splitting as

$$
\begin{aligned}
D_{\mathrm{Lie}}= & \sum_{\sigma} \mu_{\mathrm{Lie}}(\sigma) \sum_{x, y \in \Lambda} \frac{q\left(\sigma, \sigma^{x, y}\right)}{q\left(\sigma^{x, y}, \sigma\right)} C_{\mathrm{Lie}}\left(\sigma^{x, y}, \sigma\right) \\
& +\sum_{x, y, z, w \in \Lambda} \mu_{\mathrm{Lie}}(\sigma) L_{\mathrm{Lie}}^{2}\left(\sigma, \sigma^{x, y, z, w}\right) \operatorname{atanh}\left(M_{\mathrm{Lie}}\left(\sigma^{x, y, z, w}, \sigma\right)\right) .
\end{aligned}
$$

Since the commutator $C_{\text {Lie }}$ is zero for all choices of lattice sites but those at the 
boundaries between sub-lattices, $\partial \Lambda$, Equation (D.2) is actually

$$
\begin{aligned}
D_{\mathrm{Lie}}= & \sum_{\sigma} \mu_{\mathrm{Lie}}(\sigma) \sum_{x, y \in \partial \Lambda} \frac{q\left(\sigma, \sigma^{x, y}\right)}{q\left(\sigma^{x, y}, \sigma\right)} C_{\mathrm{Lie}}\left(\sigma^{x, y}, \sigma\right) \\
& +\sum_{x, y, z, w \in \partial \Lambda} \mu_{\mathrm{Lie}}(\sigma) L_{\mathrm{Lie}}^{2}\left(\sigma, \sigma^{x, y, z, w}\right) \operatorname{atanh}\left(M_{\mathrm{Lie}}\left(\sigma^{x, y, z, w}, \sigma\right)\right) .
\end{aligned}
$$

Therefore, for nearest neighbor interactions in a square $N \times N$ lattice, the coefficient in (D.3) has cost of computation $O\left(N^{2}\right)$. Note that the difference in scaling of the cost is because of the underlying diffusion dynamics and which imply that $d\left(\sigma, \sigma^{\prime}\right)=1$, that is, the states the system can reach in one step from $\sigma$, are precisely $\sigma^{\prime}=\sigma^{x, y}$ for $x, y$ distinct lattice sites. However, estimating coefficient (D.3) is more of a diagnostic that does not have to be computed while simulating the large system, which is why we normalize coefficients by their scaling while estimating. 